\title{
Generalized Coordinated Port Selection in a Multi-Cell Distributed Antenna System using Semidefinite Relaxation
}

\author{
Gurhan Bulu*, Talha Ahmad ${ }^{\dagger}$, Ramy Gohary ${ }^{\ddagger}$, Halim Yanikomeroglu ${ }^{\ddagger}$, Cenk Toker* \\ * Department of Electrical and Electronics Engineering, Hacettepe University, Ankara Turkey \\ $\dagger$ Ericsson Canada, Ottawa, Ontario, Canada \\ $\ddagger$ Department of Systems and Computer Engineering, Carleton University, Ottawa, Ontario, Canada \\ Email: \{bulu, cenk.toker\}@ee.hacettepe.edu.tr, talha.ahmad@ericsson.com, \{gohary, halim\}@ sce.carleton.ca
}

\begin{abstract}
The downlink of a coordinated multi-cell distributed antenna system is considered. In [1], coordinated port selection was shown to achieve significant performance gains. However, in the system considered therein, the ports in each cell were constrained to transmit only to user terminals (UTs) in that cell. In this work, we consider a generalization of the problem considered in [1] by alleviating this constraint. We formulate the problem of determining the ports that maximize the minimum signal to interference plus noise ratio observed by the UTs as a binary-constrained optimization problem. Observing that it is NP-hard, we propose a semidefinite relaxation and Gaussian randomization based technique to obtain close-to-optimal solutions. Our simulation results show that the performance achieved by the proposed technique approaches that of the optimal solution. It is also shown that the proposed technique outperforms the one in [1], particularly for cell-edge UTs, albeit with an increased computational complexity.
\end{abstract}

Index Terms-Distributed antenna system, coordinated port selection, semidefinite relaxation, Gaussian randomization.

\section{INTRODUCTION}

Multi-antenna wireless communication systems can yield significant performance improvements. However, systems in which the transmit antennas are collocated result in significant attenuation experienced by cell-edge user terminals (UTs). A candidate solution to improve the signal to interference plus noise ratios (SINRs) is to disperse the antennas over the coverage area and use a coordinated multi-point strategy.

Distributed antennas have been shown to increase the coverage and the capacity of both indoor wireless and cellular systems [2], [3], [4]. In [5] \& [6], it was shown that to realize the potential gains of such systems, proper selection of the available antenna ports is necessary. In [1] \& [7], a novel coordinated port selection technique was proposed for maximizing the minimum SINR observed by the UTs in the downlink of a multi-cell distributed antenna system. However, in the system considered therein, the antenna ports in each cell were constrained to transmit only to UTs in that cell.

In this paper, we consider a system that is a generalization of the one in [1] \& [7]. In particular, we consider a cluster of cells in which the base stations (BSs) transmit in a coordinated manner. A set of distributed antenna ports is available in each cell, in which a particular frequency-time resource block (RB) is assigned to at most one UT. Each port uses a fixed transmit power, and unlike [1] \& [7], can transmit to any UT in the cluster. Our goal is to improve performance by selecting the ports that maximize the minimum SINR observed by the UTs. This generalized port selection problem is cast as a binaryconstrained optimization problem. Observing that it is NPhard [8], we use semidefinite relaxation (SDR) and Gaussian randomization to obtain candidate solutions to the original problem [9]. Simulation results suggest that the proposed technique yields close-to-optimal solutions, and also outperforms that proposed in [1] \& [7], particularly for cell-edge UTs.

\section{SySTEM MODEL}

The focus in this paper is port selection in a multicell distributed antenna system (in which interference is a concern) in the context of one single RB. The consideration of multiple RBs with some fairness measure, and thus the integration of resource allocation and port selection, will be an important (yet not straightforward) extension.

In this work, we consider a cluster of $M$ cells with a single $\mathrm{RB}$. There is one UT in each cell; therefore, each RB is reused in all cells. The BS in each cell is connected to $L$ distributed single-antenna ports with high-speed links (see Fig. 1). The transmissions of the BSs in the cluster are coordinated by a central network entity, which has reliable knowledge of the gains between the $L M$ ports and each UT in the cluster. In contrast to [1] \& [7], each of the $M$ single-antenna UTs can be served by any combination of the $L M$ antenna ports in the cluster. However, each port can transmit to at most one UT (due to the single antenna constraint).

Let $\Gamma \in\{0,1\}^{L M \times M}$ be a matrix that represents the mapping between the ports and the UTs. In particular, if the $i m$-th entry of $\boldsymbol{\Gamma}, \gamma_{i m}$, is equal to one, this means that the $i$-th port is used to serve the UT in the $m$-th cell. Furthermore, to restrict each port to transmit to at most a single UT, $\sum_{m=1}^{M} \boldsymbol{\Gamma}_{i, m} \leq 1$, for $i=1, \ldots, L M$. Let $w_{i}$ and $P_{i}$ denote the complex beam steering coefficient and fixed transmit power of the $i$-th port, respectively. Then the received signal of the UT 


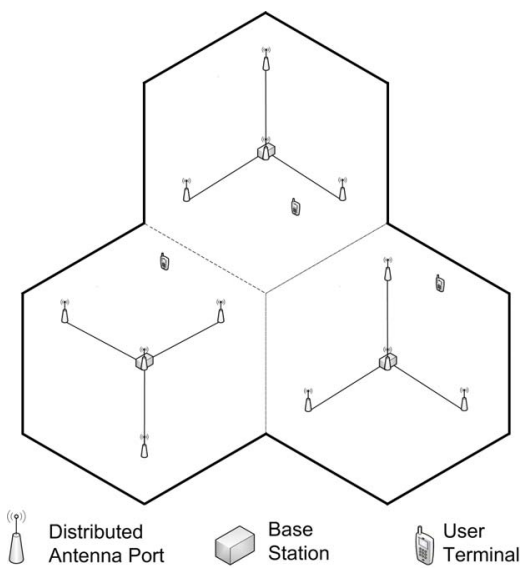

Fig. 1: A three-cell cluster with four ports per cell.

in the $m$-th cell is given by

$$
\begin{gathered}
y_{m}=\sum_{i=1}^{L M} \gamma_{i m} \sqrt{P_{i}} w_{i} h_{i m} x_{m}+ \\
\sum_{n=1, n \neq m}^{M} \sum_{j=1}^{L M} \gamma_{j n} \sqrt{P_{j}} w_{j} h_{j m} x_{n}+\eta_{m}, \quad \forall m,
\end{gathered}
$$

where, $h_{i m}$ is the complex-valued channel gain between the $i$-th port and the UT in the $m$-th cell, $x_{m}$ is the normalized data symbol of this UT, satisfying $E\left\{x_{m} x_{n}\right\}=\delta_{m n}$, and $\eta_{m} \sim \mathcal{C N}\left(0, \sigma^{2}\right)$ represents its additive white Gaussian noise.

Let $\boldsymbol{w}$ be a vector containing the complex beam steering coefficients of the $L M$ ports, $\left\{w_{i}\right\}$, and let $\gamma \in\{0,1\}^{L M^{2} \times 1}$ be a vectorized representation of $\boldsymbol{\Gamma}$, i.e., $\gamma=\operatorname{vec}(\boldsymbol{\Gamma})$. As such, the SINR of the UT in the $m$-th cell can be expressed as

$$
\operatorname{SINR}_{m}(\boldsymbol{\gamma}, \boldsymbol{w})=\frac{\left|\sum_{i=1}^{L M} \gamma_{i m} \sqrt{P_{i}} w_{i} h_{i m}\right|^{2}}{\sum_{n=1, n \neq m}^{M}\left|\sum_{i=1}^{L M} \gamma_{i n} \sqrt{P_{i}} w_{i} h_{i m}\right|^{2}+\sigma^{2}} .
$$

\section{Coordinated Port Selection}

The main idea of using a distributed, instead of co-located, antenna system is to improve the quality of service to all UTs, including the cell-edge ones. Such a goal can be attained by selecting the ports and the corresponding beam steering coefficients that jointly maximize the minimum SINR. These ports and the coefficients can be obtained by solving the following optimization problem:

$$
\max _{\boldsymbol{\gamma}, \boldsymbol{w}} \min _{m} \operatorname{SINR}_{m}(\boldsymbol{\gamma}, \boldsymbol{w}),
$$

subject to $\quad 0 \leq \sum_{m=1}^{M}[\gamma]_{i+(m-1) L M} \leq 1, \quad i=1, \ldots, L M$,

$$
\gamma \in\{0,1\}^{L M^{2} \times 1}
$$

This problem is non-convex and difficult to solve jointly for $\gamma$ and $\boldsymbol{w}$. In fact, for any given $\gamma$, finding the optimal $\boldsymbol{w}$, satisfying (3c) is NP-hard [4]. Conversely, for any given $\boldsymbol{w}$, finding the optimal $\gamma$, satisfying (3b) is also NP-hard [8]. Our focus herein is on the latter of these two problems, which can be cast as an optimization problem that is amenable to techniques that efficiently yield close-to-optimal solutions.

In this paper, we chose the beam-steering coefficients (denoted by $\boldsymbol{w}_{\mathbf{0}}$ ) as complex numbers with unity magnitude and with phases conjugate of the phases of the channels between ports and their intended UTs. As such, the signals received from multiple ports at an intended UT are phase-aligned. Note that this is not necessarily the optimal strategy, as the chosen coefficients only maximizes the numerator of the SINR expression without any attempt of minimizing the interference component. With these beam-steering coefficients our goal in this section is to obtain the set of ports that maximizes the minimum SINR observed by the UTs in the cluster. In the rest of this paper, we use the term "conditional optimum" to emphasize the fact that the optimum solution is conditioned under the chosen beam-steering coefficients. To facilitate the optimization technique used herein, the SINR of the UT in the $m$-th cell can be expressed as

$$
\operatorname{SINR}_{m}(\boldsymbol{\gamma}, \boldsymbol{w})=\frac{\boldsymbol{\gamma}^{T} \boldsymbol{A}_{m} \boldsymbol{\gamma}}{\boldsymbol{\gamma}^{T} \boldsymbol{B}_{m} \boldsymbol{\gamma}+\sigma^{2}},
$$

where $\boldsymbol{A}_{m} \in \mathbb{R}^{L M^{2} \times L M^{2}}$ and $\boldsymbol{B}_{m} \in \mathbb{R}^{L M^{2} \times L M^{2}}$ are blockdiagonal matrices defined as

$$
\begin{aligned}
& \boldsymbol{A}_{m}=\oplus_{n=1}^{m-1} \mathbf{0}_{L M} \oplus \boldsymbol{D}_{m} \oplus_{n=m+1}^{M} \mathbf{0}_{L M}, \text { and } \\
& \boldsymbol{B}_{m}=\oplus_{n=1}^{m-1} \boldsymbol{D}_{n} \oplus \mathbf{0}_{L M} \oplus_{n=m+1}^{M} \boldsymbol{D}_{n},
\end{aligned}
$$

where $\oplus$ denotes the direct sum operation, $\mathbf{0}_{L M}$ is an $L M \times$ $L M$ all-zero matrix , and the $i j$-th entry of the matrix $\boldsymbol{D}_{m} \in$ $\mathbb{R}^{L M \times L M}$ is given by $\left[\boldsymbol{D}_{m}\right]_{i j}=\sqrt{P_{i} P_{j}} \operatorname{Re}\left\{h_{i m} w_{i} w_{j}^{*} h_{j m}^{*}\right\}$. Using this notation, the optimization problem corresponding to (3) for selecting the SINR maximizing set of ports with the given $\boldsymbol{w}_{\mathbf{0}}$ can be expressed as

$$
\begin{aligned}
\max _{\boldsymbol{\gamma}} \min _{m} & \frac{\boldsymbol{\gamma}^{T} \boldsymbol{A}_{m} \boldsymbol{\gamma}}{\boldsymbol{\gamma}^{T} \boldsymbol{B}_{m} \boldsymbol{\gamma}+\sigma^{2}}, \\
\text { subject to } & 0 \leq \sum_{m=1}^{M}[\boldsymbol{\gamma}]_{i+(m-1) L M} \leq 1, \quad i=1, \ldots, L M, \\
& \gamma \in\{0,1\}^{L M^{2} \times 1} .
\end{aligned}
$$

This is a binary-constrained optimization problem, which can be shown to be NP-hard. To find a close-to-optimal solution for this problem, we will use SDR. To use this technique, we will reformulate the problem in (7) in a manner similar to that in [1]. In particular, we first introduce the vector $\psi=2 \gamma-1$, where $\psi \in\{-1,1\}^{L M^{2} \times 1}, \mathbf{1}$ denotes the all-one vector, and

$$
\gamma=\frac{1}{2}(\psi+1) \text {. }
$$


Substituting (8) into (7), the problem can be rewritten as

$$
\begin{array}{cc}
\max _{\boldsymbol{\psi}} \min _{m} \frac{\boldsymbol{\psi}^{T} \boldsymbol{A}_{m} \boldsymbol{\psi}+2 \boldsymbol{\psi}^{T} \boldsymbol{A}_{m} \mathbf{1}+\mathbf{1}^{T} \boldsymbol{A}_{m} \mathbf{1}}{\boldsymbol{\psi}^{T} \boldsymbol{B}_{m} \boldsymbol{\psi}+2 \boldsymbol{\psi}^{T} \boldsymbol{B}_{m} \mathbf{1}+\mathbf{1}^{T} \boldsymbol{B}_{m} \mathbf{1}+4 \sigma^{2}}, \\
\text { subject to } & -M \leq \sum_{m=1}^{M}[\boldsymbol{\psi}]_{i+(m-1) L M} \leq 2-M, \\
& \quad i=1, \ldots, L M, \\
& \boldsymbol{\psi} \in\{-1,1\}^{L M^{2} \times 1} .
\end{array}
$$

Next, we cast the non-homogeneous quadratic forms in the numerator and denominator of (9a) in the homogeneous forms

$$
\left[\begin{array}{ll}
\boldsymbol{\psi}^{T} & 1]
\end{array}\right] \boldsymbol{E}_{m}\left[\boldsymbol{\psi}^{T} 1\right]^{T} \text {, and }\left[\boldsymbol{\psi}^{T} 1\right] \boldsymbol{F}_{m}\left[\boldsymbol{\psi}^{T} 1\right]^{T},
$$

respectively, where

$$
\begin{aligned}
& \boldsymbol{E}_{m}=\left[\begin{array}{cc}
\boldsymbol{A}_{m} & \boldsymbol{A}_{m} \mathbf{1} \\
\mathbf{1}^{T} \boldsymbol{A}_{m} & \mathbf{1}^{T} \boldsymbol{A}_{m} \mathbf{1}
\end{array}\right], \text { and } \\
& \boldsymbol{F}_{m}=\left[\begin{array}{cc}
\boldsymbol{B}_{m} & \boldsymbol{B}_{m} \mathbf{1} \\
\mathbf{1}^{T} \boldsymbol{B}_{m} & \mathbf{1}^{T} \boldsymbol{B}_{m} \mathbf{1}+4 \sigma^{2}
\end{array}\right] .
\end{aligned}
$$

Using this form and letting $\boldsymbol{\Phi}=\boldsymbol{\psi} \boldsymbol{\psi}^{T}$, the problem in (9) can be expressed as

$$
\begin{aligned}
& \max _{\boldsymbol{\Phi}, \boldsymbol{\psi}} \min _{m} \frac{\operatorname{Tr}\left(\boldsymbol{E}_{m}\left[\begin{array}{cc}
\boldsymbol{\Phi} & \boldsymbol{\psi} \\
\boldsymbol{\psi}^{T} & 1
\end{array}\right]\right)}{\operatorname{Tr}\left(\boldsymbol{F}_{m}\left[\begin{array}{cc}
\boldsymbol{\Phi} & \boldsymbol{\psi} \\
\boldsymbol{\psi}^{T} & 1
\end{array}\right]\right)}, \\
& \text { subject to } \boldsymbol{\Phi}-\boldsymbol{\psi} \boldsymbol{\psi}^{T}=\mathbf{0} \text {, } \\
& \operatorname{diag}(\boldsymbol{\Phi})=\mathbf{1}, \\
& -M \leq \sum_{m=1}^{M}[\boldsymbol{\psi}]_{i+(m-1) L M} \leq 2-M, \\
& i=1, \ldots, L M \\
& \sum_{m=1}^{M}[\boldsymbol{\Phi}]_{j, i+(m-1) L M} \leq|2-M|, \\
& i=1, \ldots, L M, j=1, \ldots, L M^{2} .
\end{aligned}
$$

In this formulation, the binary constraint in $(9 \mathrm{c})$ is replaced with the equivalent linear constraint in $(10 \mathrm{c})$, and the constraint in (10e) is obtained from (9b) and (9c).

\section{A. Positive Semidefinite Relaxation}

The constraint in (10b) imposes a non-convex rank-1 constraint on $\boldsymbol{\Phi}$, thereby resulting in the NP-hardness of the optimization problem in (10). To obtain a close-to-optimal solution of this problem we consider a relaxed version of (10) in which the equality constraint in $(10 \mathrm{~b})$ is replaced with the generalized matrix inequality

$$
\boldsymbol{X}-\boldsymbol{x} \boldsymbol{x}^{T} \succeq \mathbf{0},
$$

where $\boldsymbol{X}$ and $\boldsymbol{x}$ correspond to $\boldsymbol{\Phi}$ and $\boldsymbol{\psi}$ in the original problem, respectively. Note that this constraint is non-convex. Also, the matrix $\boldsymbol{X}-\boldsymbol{x} \boldsymbol{x}^{T}$ is the Schur complement of

$$
\boldsymbol{\Omega}=\left[\begin{array}{cc}
\boldsymbol{X} & \boldsymbol{x} \\
\boldsymbol{x}^{T} & 1
\end{array}\right],
$$

and is positive semidefinite (PSD) if and only if $\Omega$ is PSD. Using this observation, the constraint in (11) can be expressed as a convex linear matrix inequality constraint.

It can be seen that the objective function of the relaxed problem is also non-convex. Introducing an auxiliary variable, $t$, which lower-bounds the objective function, the relaxed problem can be written as

$$
\begin{array}{rl}
\max _{t, \boldsymbol{X}, \boldsymbol{x}} & t, \\
\text { subject to } & t \leq \frac{\operatorname{Tr}\left(\boldsymbol{E}_{m} \boldsymbol{\Omega}\right)}{\operatorname{Tr}\left(\boldsymbol{F}_{m} \boldsymbol{\Omega}\right)}, \quad \forall m, \\
& \boldsymbol{\Omega}=\left[\begin{array}{cc}
\boldsymbol{X} & \boldsymbol{x} \\
\boldsymbol{x}^{T} & 1
\end{array}\right] \succeq \mathbf{0}, \\
& \operatorname{diag}(\boldsymbol{X})=\mathbf{1}, \\
& -M \leq \sum_{m=1}^{M}[\boldsymbol{x}]_{i+(m-1) L M} \leq 2-M, \\
& \sum_{m=1}^{M}[\boldsymbol{X}]_{j, i+(m-1) L M} \leq|2-M|, \quad i, L M, \\
& i=1, \ldots, L M, j=1, \ldots, L M^{2} .
\end{array}
$$

The inequality constraint in (12b) involves products of the form $t \boldsymbol{x}$ and $t \boldsymbol{X}$, which implies that the problem in (12) is still non-convex in $(t, \boldsymbol{x}, \boldsymbol{X})$. However, analogous to [1], we can exploit the quasi-linearity of the constraints to iteratively solve the problem using a series of convex feasibility problems (see [10, Section 4.2.5]). This approach is based on bisection search, wherein the SINR is upper-bounded by $t_{\text {max }}=\min _{m=1 \ldots, M} \tilde{\boldsymbol{x}}_{m}^{T} \boldsymbol{A}_{m} \tilde{\boldsymbol{x}}_{m} / \sigma^{2}$, where

$$
\tilde{\boldsymbol{x}}_{m}=\overbrace{-1, \ldots,-1}^{(m-1) L M}, \overbrace{1, \ldots, 1}^{L M}, \overbrace{-1, \ldots,-1}^{(M-m) L M}] .
$$

\section{B. Randomization for Coordinated Port Selection}

In this section, we use the optimal solution of (12), denoted by $\boldsymbol{X}^{\star}$ and $\boldsymbol{x}^{\star}$, to generate candidate solutions of (9). To do so, we use the Gaussian randomization technique, wherein a set of $J$ random vectors $\mathcal{V}=\left\{\boldsymbol{v}^{(j)}\right\}_{j=1}^{J}$, where $\boldsymbol{v}^{(j)} \in$ $\mathbb{R}^{L M^{2} \times 1}$ for all $j$, is generated from the Gaussian distribution $\mathcal{N}\left(\boldsymbol{x}^{\star}, \boldsymbol{X}^{\star}-\boldsymbol{x}^{\star} \boldsymbol{x}^{\star T}\right)$. It can be seen that for sufficiently large $J$, the vectors in $\mathcal{V}$ provide an approximate solution to the following stochastic optimization problem:

$$
\max _{\substack{\boldsymbol{X}^{\star}=\mathrm{E}\left\{\boldsymbol{v} \boldsymbol{v}^{T}\right\} \\ \boldsymbol{x}^{\star}=\mathrm{E}\{\boldsymbol{v}\}}} \quad t,
$$

subject to

$$
\begin{aligned}
& \mathrm{E}\left\{\left[\begin{array}{ll}
\boldsymbol{v}^{T} & 1
\end{array}\right]\left(t \boldsymbol{F}_{m}-\boldsymbol{E}_{m}\right)\left[\begin{array}{ll}
\boldsymbol{v}^{T} & 1
\end{array}\right]^{T}\right\} \leq 0, \forall m \\
& \mathrm{E}\left\{[\boldsymbol{v}]_{r}^{2}\right\}=1, \quad r=1, \ldots, L M .
\end{aligned}
$$

Using (11), it can be seen that this optimization problem is equivalent to the one in (12), except (12e) and (12f) [9]. To satisfy these remaining constraints, for each $\boldsymbol{v}^{(k)} \in \mathcal{V}$, we first 
normalize each element of $\boldsymbol{v}^{(k)}$ as follows:

$$
\left[\boldsymbol{z}^{(k)}\right]_{j}=\frac{\left[\boldsymbol{v}^{(k)}-\boldsymbol{x}^{\star}\right]_{j}}{\left[\boldsymbol{X}^{\star}\right]_{j, j}}, j=1, \ldots, L M^{2} .
$$

Next, we identify the largest association between the UTs and each of the $L M$ ports. In particular, let

$$
a_{i}^{(k)}=\max \left\{\left[\boldsymbol{z}^{(k)}\right]_{i+(m-1) L M}\right\}_{m=1, \ldots, M}, i=1, \ldots, L M .
$$

Then, we truncate the association of all other ports with each UT, such that only the port with the largest association with this UT is allowed to transmit to it. Specifically,

$$
\begin{gathered}
{\left[\boldsymbol{u}^{(k)}\right]_{i+(m-1) L M}=\left\{\begin{array}{cc}
a_{i}^{(k)}, & \text { if }\left[\boldsymbol{z}^{(k)}\right]_{i+(m-1) L M}=a_{i}^{(k)}, \\
-1, & \text { otherwise }
\end{array}\right.} \\
i=1, \ldots, L M, \quad m=1, \ldots, M .
\end{gathered}
$$

In doing so, (12e) and (12f) are satisfied. Now, to extract candidate solutions to the problem in (9), the entries of each realization of $\boldsymbol{u}^{(k)}$ are quantized. In particular, for each $\boldsymbol{u}^{(k)}$, a candidate binary solution $\psi^{(k)}$ could be obtained as

$$
\boldsymbol{\psi}^{(k)}=\operatorname{sgn}\left(\boldsymbol{u}^{(k)}\right), \quad k=1, \ldots, K,
$$

where $\operatorname{sgn}(\cdot)$ is the element-wise signum function and $K$ is the number of Gaussian samples. Next, using (8), the corresponding candidate solutions of (7) are obtained and the one yielding the largest objective is chosen; i.e.,

$$
\boldsymbol{\gamma}^{\star}=\arg \max _{k=1, \ldots, K} \min _{m=1, \ldots, M} \operatorname{SINR}_{m}\left(\boldsymbol{\gamma}^{(k)}\right) .
$$

\section{Computational Complexity Analysis}

The goal of the technique is to find the optimal port state vector. Analogous to [1], it can be shown that the computational complexity of the proposed technique is upperbounded by

$$
O\left(\left(L M^{2}\right)^{4.5} \log (1 / \epsilon) \log \left(t_{\max } / \epsilon\right)+\left(L M^{2}\right)^{2} J\right),
$$

where $\epsilon>0$ is the solution accuracy. The port state vector could also be found by exhaustive search over all possible vectors. The computational complexity of this approach is $O\left(2^{L M^{2}}\right)$, and it is inefficient for large $L$ and $M$.

\section{Performance Evaluation}

We use Monte Carlo simulation to measure the performance of the proposed algorithm. The setup used for the simulations consists of $M$ hexagonal cells, each with circumradius $r_{c}$. There are four ports in each BS, i.e., $L=4$. Three of these ports are located uniformly at a distance of $\frac{2}{3} r_{c}$ from the BS at the center of the cell, and the fourth port is co-located with the BS. Such a system is illustrated in Fig. 1 with $M=3$. All the ports transmit at a fixed power $P$. In every iteration, each of the $M$ UTs is dropped randomly within each cell.

The channel model considered for the simulation is the one used in [1]. In particular, each complex channel gain is expressed as $h_{i m}=\sqrt{\rho\left(d_{i m}\right) s_{i m}} h_{i m}^{\prime}$, where $\rho(\cdot)$ is a path gain expression, $d_{i m}$ is the distance between the $i$-th port of the cluster and the UT in the $m$-th cell, $s_{i m}$ represents log-normal shadowing with $0 \mathrm{~dB}$ mean and $\sigma_{s} \mathrm{~dB}$ standard deviation, and $h_{i m}^{\prime}$ represents Rayleigh fading.

The distance values and the channel model parameters used for the simulations have been chosen from the suburban macro-cell (SMa) scenario in IMT-Advanced, and are summarized in Table I [11], [12]. The path loss channel model is chosen to be the non-line-of-sight one in [11]; using the values in Table I, $\rho\left(d_{i m}\right)$ can be expressed as $10^{-1.866-4.032 \log _{10}\left(d_{i m}\right)}$.

TABLE I: Simulation parameters for SMa

\begin{tabular}{|c|c|}
\hline Parameter & Value \\
\hline Carrier frequency $\left(f_{c}\right)$ & $2 \mathrm{GHz}$ \\
\hline Distance between base stations & $1299 \mathrm{~m}$ \\
\hline Shadowing standard deviation $\left(\sigma_{s}\right)$ & $8 \mathrm{~dB}$ \\
\hline Noise power $\left(\sigma^{2}\right)$ & $-114 \mathrm{dBm}$ \\
\hline Elevation of antenna ports & $15 \mathrm{~m}$ \\
\hline Elevation of UTs & $1.5 \mathrm{~m}$ \\
\hline
\end{tabular}

Example 1: A cluster of two cells (i.e., $M=2$ ) is considered. In Fig. 2, the largest minimum SINR that is achieved by the proposed port selection technique without the cell boundary restriction is compared with that achieved by the technique in [1], i.e., with the cell boundary restriction. It is also compared with the largest minimum SINR achieved by the conditional optimal solution. The resulting SINRs are averaged over 2000 independent channel realizations and the number of Gaussian samples, $K$, is chosen to be 500 for each iteration. The conditional optimal solution is obtained using an exhaustive search, wherein the objective function in (7a) is evaluated for all $2^{L M^{2}}$ possible port state vectors, except for those violate (7b). For the two-cell cluster considered in this example, this corresponds to searching over $2^{16}$ candidate vectors minus the ineligible ones that violate the constraint (7b).

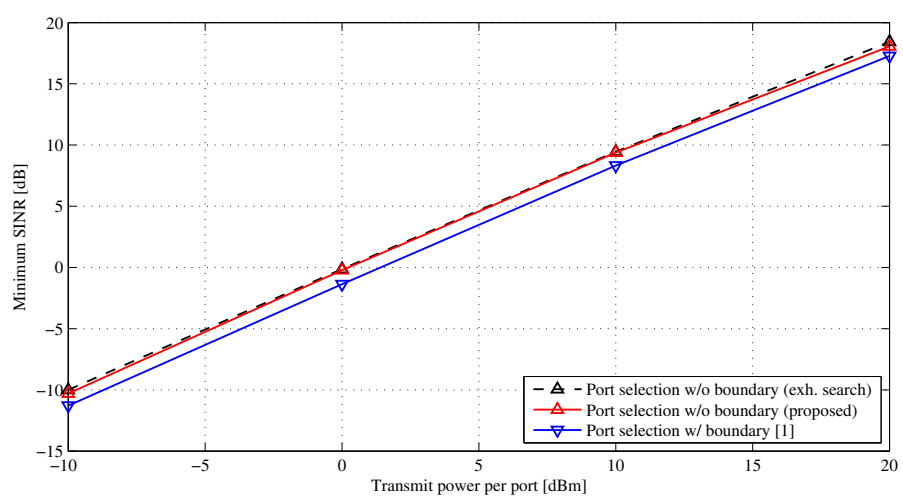

Fig. 2: A comparison between the largest minimum SINRs achieved by conditional optimal port selection, the proposed technique, and the one in [1] for a two-cell cluster.

It can be seen from Fig. 2, that the generalized technique proposed herein outperforms that in [1]. For example, at $P=10 \mathrm{dBm}$, the minimum SINR achieved by the proposed 
technique is approximately $1 \mathrm{~dB}$ higher. It can also be seen that the performance of the proposed technique approaches that of the exhaustive search based conditional optimal solution.

Example 2: In this example, a cluster of three cells is considered to further evaluate performance of the proposed technique in a more complex scenario. As such, the simulation setup contains twelve ports and each port can serve any of the UTs in the cluster. For this scenario, the conditional optimal solution is computationally prohibitive because this search involves $2^{36} \approx 6.87 \times 10^{10}$ port state vectors. In Fig. 3, analogous results to those in Fig. 2 are presented for this modified scenario. Comparing the two figures, it can be seen that the proposed technique offers a greater performance improvement for three-cell cluster. For example, at $P=10 \mathrm{dBm}$, it outperforms the technique in [1] by approximately $2 \mathrm{~dB}$.

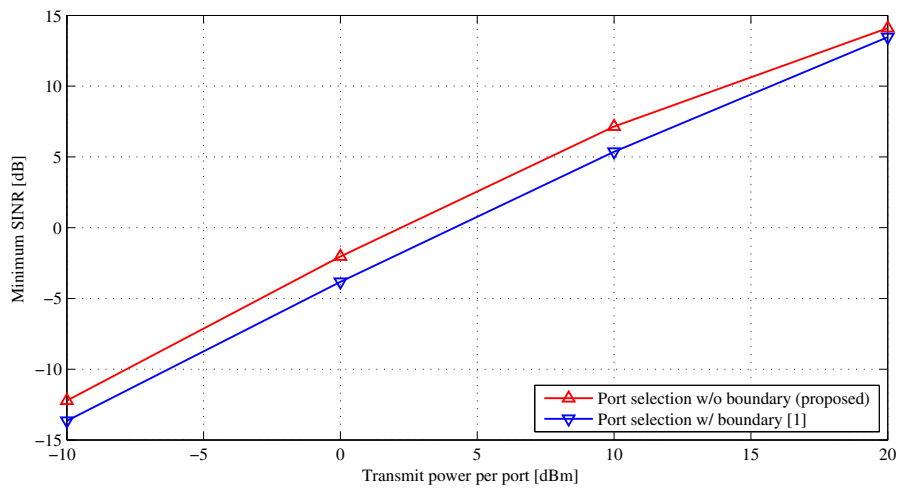

Fig. 3: A comparison between the largest minimum SINRs achieved by the proposed port selection technique and the one in [1] for a three-cell cluster.

In Fig. 4, a CDF of the SINRs achieved by all UTs is presented for the two techniques at $P=10 \mathrm{dBm}$. Comparing the SINRs at the probability value of 0.05 , it can be seen that the proposed technique outperforms the one in [1] by approximately $2.5 \mathrm{~dB}$. This corresponds to an increase of approximately 55\% in spectral efficiency according to Shannon's channel capacity theorem.

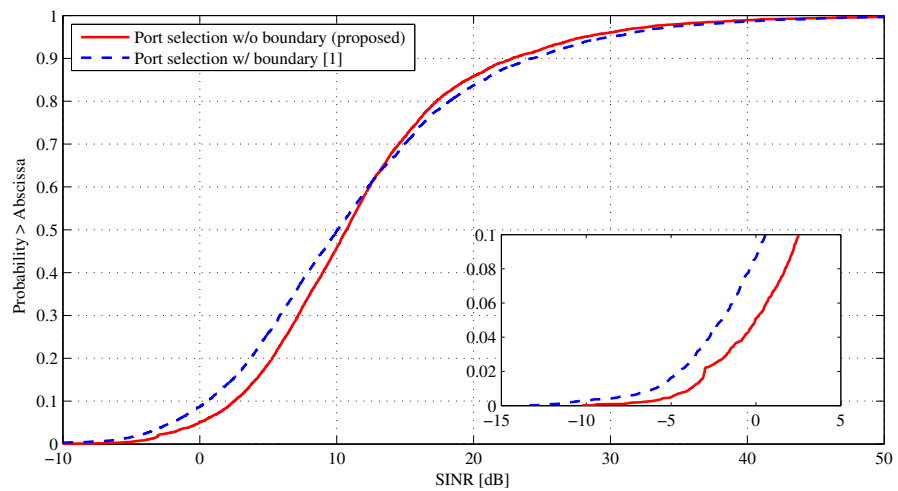

Fig. 4: CDFs of the SINR of all UTs for the proposed port selection technique and the one in [1] for a three-cell cluster at $P=10 \mathrm{dBm}$.

\section{Conclusion}

In this paper, we considered the downlink of a multicell distributed antenna system, wherein antenna ports can transmit to any UT in a cluster of coordinating cells. An SDRbased coordinated port selection technique was proposed for maximizing the minimum SINR of the UTs in the cluster. This technique was shown to yield close-to-optimal solutions. It was further shown that by removing the logical cell boundaries, the SINR of the cell edge-users is significantly increased, albeit at the expense of increased computational complexity.

\section{ACKNOWLEDGEMENT}

This work is partly supported by TUBITAK (The Scientific and Technological Research Council of Turkey), Turkey, under project no. 112E024.

\section{REFERENCES}

[1] T. Ahmad, R. Gohary, H. Yanikomeroglu, S. Al-Ahmadi, and G. Boudreau, "Coordinated port selection and beam steering optimization in a multi-cell distributed antenna system using semidefinite relaxation," IEEE Trans. Wireless Commun., vol. 11, no. 5, pp. 18611871, May 2012.

[2] A. Saleh, A. Rustako, and R. Roman, "Distributed antennas for indoor radio communications," IEEE Trans. Commun., vol. 35, no. 12, pp. 1245-1251, Dec. 1987.

[3] H. Yanikomeroglu and E. Sousa, "CDMA distributed antenna system for indoor wireless communications," in Proc. of IEEE Int. Conf. on Universal Personal Commun., vol. 2, Nov. 1993, pp. 990-994.

[4] N. Sidiropoulos, T. Davidson, and Z.-Q. Luo, "Transmit beamforming for physical-layer multicasting," IEEE Trans. Signal Process., vol. 54, no. 6, pp. 2239-2251, Jun. 2006.

[5] W. Choi and J. Andrews, "Downlink performance and capacity of distributed antenna systems in a multicell environment," IEEE Trans. Wireless Commun., vol. 6, no. 1, pp. 69-73, Jan. 2007.

[6] J. Park, E. Song, and W. Sung, "Capacity analysis for distributed antenna systems using cooperative transmission schemes in fading channels," IEEE Trans. Wireless Commun., vol. 8, no. 2, pp. 586 -592, Feb. 2009.

[7] T. Ahmad, R. Gohary, H. Yanikomeroglu, S. Al-Ahmadi, and G. Boudreau, "Coordinated max-min fair port selection in a multi-cell distributed antenna system using semidefinite relaxation," in IEEE Int. Conf. on Commun., 2012, pp. 3616-3620.

[8] L. Vandenberghe and S. Boyd, "Semidefinite programming," SIAM Rev., vol. 38, no. 1, pp. 49-95, Mar. 1996.

[9] Z.-Q. Luo, W.-K. Ma, A.-C. So, Y. Ye, and S. Zhang, "Semidefinite relaxation of quadratic optimization problems," IEEE Signal Process. Mag., vol. 27, no. 3, pp. 20-34, May 2010.

[10] S. Boyd and L. Vandenberghe, Convex Optimization. Cambridge, U.K.: Cambridge Univ. Press, 2004.

[11] International Telecommunication Union (ITU), "Guidelines for evaluation of radio interface technologies for IMT-Advanced," ITU-R, TR M.2135-1, Dec. 2009. [Online]. Available: http://www.itu.int/pub/RREP-M.2135-1-2009

[12] 3rd Generation Partnership Project (3GPP), "Evolved Universal Terrestrial Radio Access (E-UTRA); Radio Frequency (RF) system scenarios," 3GPP, TR 36.942, Dec. 2010. [Online]. Available: http://www.3gpp.org/ftp/Specs/html-info/36942.htm 